\title{
Prevalence and rates of intimate partner violence among South African women during pregnancy and the postpartum period
}

\author{
Allison K. Groves ${ }^{1}$, Dhayendre Moodley ${ }^{2}$, Luz McNaughton-Reyes ${ }^{1}$, Sandra L. Martin ${ }^{1}$, \\ Vangie Foshee $^{1}$, and Suzanne Maman ${ }^{1}$ \\ ${ }^{1}$ University of North Carolina at Chapel Hill, Chapel Hill, NC \\ ²University of KwaZulu-Natal, Durban, South Africa
}

\begin{abstract}
Objectives-Intimate partner violence (IPV) is a significant public health problem in South Africa. However, limited research exists on IPV during pregnancy and the postpartum period in South Africa. The purpose of this study is to describe the prevalence, rates and correlates of IPV among South African women during pregnancy and the first nine months postpartum.

Methods-Data are from a longitudinal study with women recruited during pregnancy between 2008 and 2010 at a public clinic in Durban. We used a modified version of the World Health Organization's IPV scale to estimate prevalence and rates of IPV during pregnancy, at four months postpartum and nine months postpartum and we used logistic regression to assess the correlates of IPV during this time.
\end{abstract}

Results-More than $20 \%$ of all women experienced at least one act of physical, psychological or sexual IPV during pregnancy. Nearly one-quarter of all women experienced at least one act of physical, psychological or sexual IPV during the first nine months postpartum. Psychological IPV was the most prevalent type of IPV during pregnancy and the first four months postpartum. Age and previous violence within the relationship were associated with IPV during pregnancy and IPV during the postpartum period.

Conclusions-The high levels of IPV during pregnancy and the postpartum period highlight the need to develop screening and intervention strategies specifically for this time. Further, women should be screened not only for physical violence but also psychological violence given that psychological violence may result in distinct negative consequences.

\section{Keywords}

intimate partner violence; pregnancy; postpartum; South Africa; prevalence; correlates

Publisher's Disclaimer: Disclaimer: The views expressed in the submitted article are our own and not an official position of the institution or funder.

Conflict of interest declaration: The authors report no conflicts of interest. 


\section{Introduction}

Intimate partner violence (IPV), defined as the experience of "physical, sexual or psychological harm by a current or former partner or spouse" (1), is a significant public health and human rights problem across the globe, and South Africa is no exception $(2,3)$. In a nationally representative study on IPV, one in three South African women had experienced physical IPV at some point in their current relationship (4). Other types of violence within intimate partnerships are also common: nearly half of all women in a crosssectional, community based study conducted in three provinces experienced emotional abuse or were denied financial support from their partner in the previous year (5). Furthermore, though the sensitivity of asking about and reporting sexual violence makes measurement notoriously difficult $(6,7)$, one national study found at least $7 \%$ of women reported being forced or persuaded to have sex against their will $(8,9)$.

IPV has significant negative health and social consequences for South African women, including injury, poor mental health, sexually transmitted infections (including HIV), substance use and death $(2,10)$. Half of all women who are killed in South Africa are killed by their intimate partners; this rate of femicide $(8.8 / 100,000)$ is the highest in the world (11). In addition, interpersonal violence, including IPV, negatively affects South African women's daily lives and accounts for $10.9 \%$ of all disability-adjusted life years (DALYs) (2).

Whereas a growing body of research has examined the prevalence of IPV in South Africa and across the globe using general samples of women, very little research has examined IPV during pregnancy and postpartum period. This gap in the literature needs attention given that IPV during pregnancy and the postpartum period may negatively affect not only women's health and well-being but that of their infants as well. To our knowledge, no research has examined the negative outcomes of IPV in the early postpartum period; however, IPV during pregnancy has been associated with physical morbidity, including preterm labor, vaginal bleeding, kidney infection, urinary tract infections and cesarean sections (12-14). IPV during pregnancy has also been associated with poor mental health, including increased emotional distress during pregnancy (15-17) and postnatal depression (18). In a retrospective case-control study in the United States where cases were defined by attempted or completed femicides and controls were randomly identified abused women living in the same city, researchers found that women who experienced IPV specifically during pregnancy were at increased risk for future femicide as compared to women who did not experience IPV specifically during pregnancy (19). Finally, infants whose mothers experience IPV during pregnancy are at greater risk of low birth weight (20-23), preterm birth (20) and perinatal death.

Even so, only two South African studies have examined IPV specifically during pregnancy. In both studies, the prevalence of physical violence was $10 \%$ or less $(5,17)$. The second study also reported that nearly $20 \%$ of women reported psychological IPV and approximately 3\% reported sexual IPV (17). To our knowledge, no studies in South Africa have examined IPV during the first nine months postpartum. 
Our understanding of correlates of IPV during pregnancy and the postpartum period in the South African context is also limited. Systematic reviews in sub-Saharan Africa and the United States suggest that age, socioeconomic status and relationship type are correlated with IPV during pregnancy, although the findings are inconsistent $(24,25)$. These reviews have also found reproductive health characteristics like pregnancy intention, parity and HIV status to be associated with IPV during pregnancy. In addition, having experienced IPV in the relationship before pregnancy has been associated with risk of IPV during pregnancy (26-28). To our knowledge, no research has examined whether these same characteristics are also associated with IPV postpartum. The purpose of this study is to examine IPV during pregnancy and the first nine months postpartum among urban South African women. In particular, we estimate the prevalence and rates of: (a) any violence; (b) physical violence; (c) psychological violence; and (d) sexual violence, we describe overlap in types of violence experienced and we assess whether demographic characteristics, reproductive health characteristics and having a history of IPV in one's relationship are associated with IPV during pregnancy or with IPV during the postpartum period

\section{Methods}

\section{Setting and study overview}

The data set for this analysis comes from women who participated in the control arm of the South Africa HIV Antenatal Post-test Support Study (SAHAPS). SAHAPS was a randomized controlled trial designed to provide women with enhanced HIV counseling and psychosocial support during pregnancy and the postpartum period. SAHAPS was conducted in a public antenatal clinic in Umlazi township (17km southwest of Durban) in the province of KwaZulu Natal (KZN). Of the nine provinces in South Africa, KZN has the highest prevalence of HIV infection, the greatest proportion of individuals living in poverty and the highest infant mortality rate (29).

Women were recruited for SAHAPS at their first antenatal visit, which on average, took place when women were $51 / 2$ months pregnant. Inclusion criteria to participate in SAHAPS were: (1) at least 18 years old, (2) had never tested for HIV or had tested negative for HIV at least 3 months prior to recruitment (3) attending first antenatal visit when HIV testing was offered (4) had a primary partner who they had been with for at least 6 months (5) planned to live in Durban for at least the next year (6) planned to bring their infant to the clinic for immunizations (7) were able to communicate in Zulu or English and (8) were not a high-risk pregnancy.

Women who consented to participate in SAHAPS completed a baseline assessment immediately after providing informed consent and prior to receiving clinical services (including HIV testing). Women also completed a behavioral assessment at four months and at nine months postpartum. Each assessment was conducted in Zulu or English by one of four trained South African survey interviewers using computer assisted personal interviews. Each interviewer completed training on conducting quantitative interviews, which included sessions on rapport building, asking sensitive questions and good ethical conduct. Women who showed signs of distress during the interview were referred to the onsite psychologist. 
The research was approved by the board of ethics at the University of North Carolina at Chapel Hill and the University of KwaZuluNatal.

\section{Sample}

A total of 747 women participated in the control arm of the SAHAPS study. To describe prevalence and rates of IPV during pregnancy, at four months postpartum and at nine months postpartum, data were used from control arm participants who were with the same partner for the entire study period (717/747), who participated in all three study assessments (468/747) and who were not missing data on any violence items (445/463), for a total sample size of 445 women. To describe correlates of IPV during pregnancy and postpartum, data were used from participants who fit the same criteria above and who were not missing data on demographic or reproductive health correlates. Logistic regression was used to examine whether IPV during pregnancy or other demographic and reproductive health characteristics were associated with study drop out at four months or nine months postpartum.

\section{Measures}

Violence was measured using a modified version of the World Health Organization (WHO) Violence Against Women instrument (30). This instrument contains thirteen items on physical, sexual and psychological violence that a woman has experienced with her current partner and has been validated in numerous South African studies (31-33). Each item has five response categories, ranging from "never" to "more than ten times."

Physical violence was measured through six items (e.g. "how many times has your current partner pushed or shoved you?") ( $a=.80$ ). Psychological violence was measured through four items (e.g. "how many times has your current partner insulted you or made you feel bad about yourself?") ( $a=.69)$. Sexual violence was measured through three items (e.g. "how many times has your current partner physically forced you to have sex when you did not want to?") ( $(\mathrm{a}=.77)$.

Items were modified in this study to reflect the specific reference period for which the women were being questioned. In the baseline survey, each item was prefaced with "during this pregnancy, how many times has your current partner..." In the four month postpartum survey, each item was prefaced with "since delivery, how many times has your current partner..." Finally, in the nine month postpartum survey, each item was prefaced with, "since the last time I saw you, how many times has your current partner..."

Binary variables were created to estimate the prevalence of physical, psychological and sexual violence during pregnancy, at four months postpartum and at nine months postpartum. Specifically, to estimate the prevalence of physical violence in pregnancy, a woman's responses across the six items on physical violence during pregnancy were summed. A woman received a 0 if she had not experienced any physical violence during pregnancy and a 1 if she had experienced one or more acts of physical violence during pregnancy. Psychological violence and sexual violence were estimated in the same fashion using the relevant violence items from the instrument. Binary variables were also created to estimate the proportion of women who had experienced (1) any violence (regardless of type) 
at each time point, (2) any violence postpartum and (3) any violence across the entire study period. Lastly, two binary variables were created to indicate whether a woman had experienced IPV in the current relationship any time before pregnancy and whether a woman had experienced IPV in the current relationship before the postpartum period.

Rate variables were created to estimate the average amount of IPV (physical, psychological, sexual and any IPV) per month that women experienced during pregnancy, between delivery and four months and between four and nine months postpartum. Women could complete each study assessment within a given window of time. For example, women's gestational ages varied at the baseline assessment because each woman enrolled in the study when she initiated antenatal care. To estimate the rate of physical violence per month during pregnancy, a woman's responses across the six items on physical violence at pregnancy were summed and then divided by her gestational age (in months).

Demographic covariates-Participants reported their age, education, and whether they currently lived with their partner at baseline. Following previous research in South Africa, we created a measure of socioeconomic status by using principal components analysis to derive a linear index from a series of asset ownership indicators and then categorized participants as belonging to the poorest $40 \%$, middle $40 \%$ or wealthiest $20 \%(34-36)$.

Reproductive health covariates-Participants reported their parity and pregnancy intention at the baseline visit. Study nurses offered participants HIV testing during pregnancy; participants who tested positive were coded as " 1 " and participants who tested negative were coded as " 0 ".

\section{Analysis}

All analyses were completed using SAS 9.4 (37). First, we calculated prevalence estimates, rates and overlap in different types of IPV for each of the three time points in the study. Next, we conducted separate bivariate analysis to examine correlates of IPV during pregnancy and of IPV postpartum. Last, we conducted multivariate logistic regression to examine whether correlates remained significantly associated with IPV during pregnancy or IPV postpartum after controlling for other variables in the model.

\section{Results}

\section{Description of sample}

Women's ages ranged from 18-44 years and almost all (94\%) had completed secondary school (see Table 1). On average, women had been in a relationship with their current partner for 4.7 years, although a majority (75\%) did not live with their partner. Thirty-six percent of women in the sample tested HIV-positive at baseline. Over one-third of women were pregnant for the first time and four-fifths described their pregnancy as unintended. On average, women were five and a half months pregnant at their first antenatal care visit (range 5-41 weeks). Attrition did not differ according to age, education, parity or with IPV experienced during pregnancy. Women who did not complete the 14 week follow up visit were significantly more likely to be in the poorest SES tercile, to live with their partner, to 
be farther along in their pregnancy at the baseline visit and to be HIV-positive than those women who completed the visit (all p's <.05). Women who did not complete the 9 month follow up visit were significantly more likely to be in the lowest SES tercile and to be HIV positive than those women who completed the visit (all p's <.05).

\section{Prevalence, rates and overlap of IPV across the entire pregnancy and postpartum period}

The IPV prevalence estimates at each time point are depicted in Figure 1. Almost half of all women (42.25\%) experienced at least one act of physical, psychological or sexual IPV (any IPV) either during pregnancy or the first nine months postpartum. Further, women experienced an average of 0.21 acts of IPV per month across pregnancy and the first nine months postpartum (range 0-5.5). While most women who experienced IPV experienced only one type of IPV within each time point; at least a third of women of experienced two or three types of IPV within each time point (Figure 2).

\section{Prevalence and rates during pregnancy}

Just over one-fifth of women (21.35\%) experienced at least one act of IPV during pregnancy, and the prevalence of psychological IPV (16.63\%) was nearly double that of physical IPV (8.76\%) and more than five times that of sexual IPV (3.15\%) (Table 2). During pregnancy, women experienced an average of 0.15 acts of IPV per month, and the average rate of psychological IPV (.09) was greater than physical IPV (.05) and sexual IPV (.02).

\section{Prevalence and rates four months postpartum}

Nearly one-quarter of women (24.94\%) experienced any IPV at least once in the first four months postpartum, and the prevalence of psychological IPV (19.55\%) was one and a half times greater than that of physical IPV (13.48\%) and ten times greater than that of sexual IPV (2.25\%). During the first four months postpartum, women experienced an average of 0.29 acts of IPV per month, and the average rate of psychological IPV (0.16) continued to be greater than physical IPV (.11) and sexual IPV (.02).

\section{Prevalence and rates between four and nine months postpartum}

Nearly one-fifth of women (17.75\%) experienced IPV at least once between four and nine months postpartum. The prevalence of psychological IPV and physical IPV were more similar to one another than at previous time points (14.38\% and $10.56 \%$, respectively) and still approximately five times greater than sexual IPV (2.47\%). Between four and nine months postpartum, women experienced an average of 0.27 acts of IPV per month and the average rates of psychological IPV and physical IPV were the same (.12) and still greater than sexual IPV (.03).

\section{Correlates of IPV during pregnancy and the postpartum period}

Results of the bivariate and multivariate regression analysis are provided in Table 3. Age was significantly associated with IPV in both pregnancy and the postpartum period ((AOR: 94, (95\% CI: 89-1.00), (AOR:.95, (.91-1.00)) such that every year increase in age reduced women's risk that they would experience IPV. Having a history of IPV in the current relationship was significantly associated with an increased risk of IPV in both pregnancy 
and postpartum ((AOR:2.73, 95\% CI:1.79, 4.60), (AOR:3.04, 95\%CI: 1.96, 4.72)), respectively. No other demographic or reproductive health characteristics were associated with IPV during this time period.

\section{Discussion}

The aims of this study were to describe the prevalence, rates and overlap of different types of IPV across pregnancy and the postpartum period, and to assess whether demographic characteristics, reproductive health characteristics and having a history of IPV in one's relationship were associated with IPV during pregnancy or with IPV during the postpartum period. Approximately $21 \%$ of all women experienced at least one act of physical, psychological or sexual IPV during pregnancy. Women's risk of IPV in the first nine months postpartum was also high: nearly one-quarter of all women experienced at least one act of physical, psychological or sexual IPV during this time. Psychological IPV was the most prevalent type during pregnancy and in the first four months postpartum. The prevalence and rates of sexual IPV were much lower than other types of IPV during pregnancy and the postpartum period. While most women who experienced IPV experienced only one type of IPV within each time point; at least a third of women experienced two or all three types of IPV within each time point. Age and previous violence within the relationship were associated with IPV during pregnancy and IPV during the postpartum period.

The prevalence of IPV during pregnancy (21.39\%) was within the range of other prevalence estimates during pregnancy in sub-Saharan Africa $(2.3 \%-57.1 \%)(5,17,38-42)$ and higher than the prevalence reported in a recent meta-analysis across thirteen studies conducted within the region $(15.23 \%$, 95\% CI 14.38-16.08\%) (25). The wide variation in prevalence is at least partially attributable to differences in measurement and definition of IPV across studies. Nonetheless, the findings demonstrate a significant proportion of South African women are at risk of IPV during pregnancy.

Intimate partner violence in the period just following childbirth has not been studied in the sub-Saharan African context. The high postpartum prevalence estimates reported across the different types of IPV in this study (estimates range from 2.0\%-24.8\%) are among the highest reported globally among populations of adult women (postpartum estimates range from $2.0 \%-7.4 \%)(27,43,44)$ and suggest that this time period is one when women are at high risk of IPV. The postpartum period can be a vulnerable time for women as they recover both physically and emotionally from pregnancy and labor. Future research should assess whether and how IPV during this time affects women's physical recovery, their ability to provide infant care, their ability to breastfeed their infant as desired and their mental health (45).

We found little evidence that demographic or reproductive health characteristics other than age were associated with IPV during this time period. Systematic reviews on these risk factors for IPV in sub-Saharan Africa and the United States have generally reported mixed findings $(24,25)$. While findings may be inconsistent due to differences in sampling, it may be that relationship and structural factors may be more important to understanding IPV during this time. Our finding that having previously experienced violence within the 
relationship was strongly associated with violence at both time points is consistent with existing literature (26-28) and supports this notion. Further research is needed to explore how other relationship and structural factors may affect women's risk of IPV during this time.

This study is not without limitations. First, the only assessment of IPV during pregnancy took place at a woman's first antenatal visit, which means that we did not ask about women's experience of IPV during the full exposure period of pregnancy. Second, the eligibility criteria for the SAHAPS study excluded women who may have been at particularly high risk for IPV, including women who were HIV positive at screening and those women who were not in stable partnerships. Third, although we attempted to maximize the disclosure of victimization through rigorous interviewer training and the use of an instrument that focused on asking behaviorally-specific questions about victimization (30), the sensitive nature of asking about IPV may have led to under-reporting. Fourth, the poorest women in our sample and HIV-positive women were significantly more likely to drop out of the study at 14 weeks and 9 months than women with more assets and HIVnegative women. Each of these potential limitations may have led us to underestimate the prevalence of IPV or may have affected our findings regarding the correlates of IPV during this time.

Nonetheless, the study has numerous strengths. To our knowledge, our study is the only one in sub-Saharan Africa to assess IPV in the postpartum period. Second, our study is one of the first studies to look at rates of different types of violence during pregnancy and the first nine months postpartum. Rates may allow for more comparability across studies because they account for differential exposure. A final strength of the study is that it gives us a better sense of the scope of IPV during pregnancy among South African women because it describes not only physical but also psychological and sexual violence women experienced during this time. Understanding the extent of psychological and sexual violence during this time period is important because both psychological IPV and sexual IPV have adverse effects that are distinct from physical IPV $(46,47)$

\section{Conclusions}

South African women are heavily burdened by IPV across the life course and the findings from this study suggest that the time during pregnancy and the postpartum period is no exception. The findings illustrate the importance of IPV screening and intervention during both the pregnancy and the postpartum period, particularly among younger women. These periods are optimal times to identify women at risk of or currently experiencing IPV as South African women are highly engaged with the health care setting during this time: $87 \%$ of South African women receive four or more antenatal sessions during their pregnancy (48). Finally, women need to be screened not only for physical violence but also for psychological violence since psychological violence was the most prevalent type of violence women experienced during pregnancy and the first four months postpartum.

South African women are not currently screened for IPV during pregnancy and the postpartum period (49), despite the fact that IPV during pregnancy was more common than 
many other conditions that women are regularly screened for during pregnancy, including preeclampsia and gestational diabetes (39). While little evidence suggests that screening alone reduces IPV, screening done in conjunction with an intervention may reduce women's risk of future IPV (50). At least four screening and support interventions (one of which was based in South Africa) have been efficacious at reducing IPV during pregnancy and/or the postpartum period (51-54). More research is needed to disseminate and evaluate the effectiveness and cost-benefits of these interventions as implemented in South African health care settings.

Interventions beyond the healthcare sector are also needed to address IPV during pregnancy and the postpartum period. While a number of community-based interventions have been effective at reducing IPV in South Africa $(55,56)$, these interventions are not specific to pregnancy and the postpartum period. Interventions tailored to meet the needs of partners of pregnant women and/or new fathers may be especially important to reduce male perpetration of violence during this time. Structural level interventions that alter norms and/or policies that facilitate the perpetration of IPV during this time are also needed.

\section{Acknowledgments}

Many thanks to each of the South African women who participated in this study for helping us to better understand IPV during pregnancy and the postpartum period.

Sources of support: This research was sponsored by the U.S. National Institute of Mental Health (R01HD050134) and an institutional training grant DHHS/NIH/NIAID T32 AI007001-34. We also received funds from the Open Society Institute and Elton John Foundation (20020472/20030878).

\section{References}

1. CDC. [Accessed January 10 2011] Intimate Partner Violence: Definitions. 2010. http:// www.cdc.gov/ViolencePrevention/intimatepartnerviolence/definitions.html.

2. Norman R, Schneider M, Bradshaw D, et al. Interpersonal violence: an important risk factor for disease and injury in South Africa. Population Health Metrics. 2010; 8(1):32. [PubMed: 21118578]

3. Organization WH. Global and regional estimates of violence against women: prevalence and health effects of intimate partner violence and non-partner sexual violence: World Health Organization. 2013.

4. Gass JD, Stein DJ, Williams DR, et al. Intimate partner violence, health behaviours, and chronic physical illness among South African women. SAMJ: South African Medical Journal. 2010; 100(9): 582-585.

5. Jewkes R, Penn-Kekana L, Levin J, et al. Prevalence of emotional, physical and sexual abuse of women in three South African provinces. South Africa Medical Journal. 2001; 91(5):421-428.

6. Ellsberg M, Heise L, Peña R, et al. Researching Domestic Violence Against Women: Methodological and Ethical Considerations. Studies in Family Planning. 2001; 32(1):1-16. [PubMed: 11326453]

7. Koss MP. Detecting the Scope of Rape A Review of Prevalence Research Methods. Journal of interpersonal violence. 1993; 8(2):198-222.

8. Jewkes R, Abrahams N. The epidemiology of rape and sexual coercion in South Africa: an overview. Social Science \& Medicine. 2002; 55(7):1231-1244. [PubMed: 12365533]

9. Demographic and Health Survey. Pretoria: Department of Health. 1998

10. Jewkes R, Morrell R. Gender and sexuality: emerging perspectives from the heterosexual epidemic in South Africa and implications for HIV risk and prevention. Journal of the International AIDS Society. 2010; 13(1):6. [PubMed: 20181124] 
11. Abrahams N, Jewkes R, Martin LJ, et al. Mortality of women from intimate partner violence in South Africa: a national epidemiological study. Violence and victims. 2009; 24(4):546-556. [PubMed: 19694357]

12. Sharps PW, Laughon K, Giangrande SK. Intimate Partner Violence and the Childbearing Year Maternal and Infant Health Consequences. Trauma, Violence, \& Abuse. 2007; 8(2):105-116.

13. Silverman JG, Decker MR, Reed E, et al. Intimate partner violence victimization prior to and during pregnancy among women residing in 26 US states: associations with maternal and neonatal health. American journal of obstetrics and gynecology. 2006; 195(1):140. [PubMed: 16813751]

14. Hassan M, Kashanian M, Hassan M, et al. Maternal outcomes of intimate partner violence during pregnancy: study in Iran. Public health. 2014

15. Lancaster CA, Gold KJ, Flynn HA, et al. Risk factors for depressive symptoms during pregnancy: a systematic review. American journal of obstetrics and gynecology. 2010; 202(1):5-14. [PubMed: 20096252]

16. Fisher J, Tran T, Kriitmaa K, et al. Common perinatal mental disorders in northern Viet Nam: community prevalence and health care use. Bulletin of the World Health Organization. 2010; 88(10):737-745. [PubMed: 20931058]

17. Groves AK, Kagee A, Maman S, et al. Associations between intimate partner violence and emotional distress among pregnant women in Durban, South Africa. Journal of interpersonal violence. 2012; 27(7):1341-1356. [PubMed: 22203635]

18. Ludermir AB, Lewis G, Valongueiro SA, et al. Violence against women by their intimate partner during pregnancy and postnatal depression: a prospective cohort study. The Lancet. 2010; 376(9744):903-910.

19. McFarlane J, Campbell JC, Sharps P, et al. Abuse during pregnancy and femicide: urgent implications for women's health. Obstetrics \& Gynecology. 2002; 100(1):27-36. [PubMed: 12100800]

20. Coker AL, Sanderson M, Dong B. Partner violence during pregnancy and risk of adverse pregnancy outcomes. Paediatric and perinatal epidemiology. 2004; 18(4):260-269. [PubMed: 15255879]

21. Valladares E, Ellsberg M, Peña R, et al. Physical partner abuse during pregnancy: a risk factor for low birth weight in Nicaragua. Obstetrics \& Gynecology. 2002; 100(4):700-705. [PubMed: 12383537]

22. Murphy CC, Schei B, Myhr TL, et al. Abuse: a risk factor for low birth weight? A systematic review and meta-analysis. Canadian Medical Association Journal. 2001; 164(11):1567-1572. [PubMed: 11402794]

23. Koen N, Wyatt GE, Williams JK, et al. Intimate partner violence: associations with low infant birthweight in a South African birth cohort. Metab Brain Dis. 2014:1-19. [PubMed: 24307179]

24. Taillieu TL, Brownridge DA. Violence against pregnant women: Prevalence, patterns, risk factors, theories, and directions for future research. Aggression and Violent Behavior. 2010; 15(1):14-35.

25. Shamu S, Abrahams N, Temmerman M, et al. A systematic review of African studies on intimate partner violence against pregnant women: prevalence and risk factors. PloS one. 2011; 6(3):e17591. [PubMed: 21408120]

26. Stewart DE, Cecutti A. Physical abuse in pregnancy. CMAJ: Canadian Medical Association Journal. 1993; 149(9):1257.

27. Martin SL, Mackie L, Kupper LL, et al. Physical abuse of women before, during, and after pregnancy. JAMA: the journal of the American Medical Association. 2001; 285(12):1581-1584.

28. Olagbuji B, Ezeanochie M, Ande A, et al. Trends and determinants of pregnancy-related domestic violence in a referral center in southern Nigeria. International Journal of Gynecology \& Obstetrics. 2010; 108(2):101-103. [PubMed: 19939379]

29. Coovadia H, Jewkes R, Barron P, et al. The health and health system of South Africa: historical roots of current public health challenges. The Lancet. 2009; 374(9692):817-834.

30. Garcia-Moreno, C.; Jansen, HAFM.; Ellsberg, M., et al. WHO multi-country study on women's health and domestic violence against women: initial results on prevalence, health outcomes and women's responses: World Health Organization. 2005. 
31. Dunkle KL, Jewkes RK, Brown HC, et al. Gender-based violence, relationship power, and risk of HIV infection in women attending antenatal clinics in South Africa. The lancet. 2004

32. Jewkes R, Dunkle K, Nduna M, et al. Factors associated with HIV sero-status in young rural South African women: connections between intimate partner violence and HIV. International Journal of Epidemiology. 2006; 35(6):1461-1468. [PubMed: 17008362]

33. Jewkes RK, Dunkle K, Nduna M, et al. Intimate partner violence, relationship power inequity, and incidence of HIV infection in young women in South Africa: a cohort study. The Lancet. 2010; 376(9734):41-48.

34. Filmer D, Pritchett LH. Estimating wealth effects without expenditure Data-Or tears: An application to educational enrollments in states of india*. Demography. 2001; 38(1):115-132. [PubMed: 11227840]

35. Bärnighausen T, Hosegood V, Timaeus IM, et al. The socioeconomic determinants of HIV incidence: evidence from a longitudinal, population-based study in rural South Africa. AIDS (London, England). 2007; 21(Suppl 7):S29.

36. Blaauw, D.; Penn-Kekana, L., editors. Socio-economic inequalities and maternal health in South Africa; Exposé présenté à la 22e Conference on Priorities in Perinatal Care in South Africa; 2003.

37. SAS. SAS version 9.2. Cary, NC: SAS Institute; 2009.

38. Abasiubong F, Abasiattai AM, Bassey EA, et al. Demographic risk factors in domestic violence among pregnant women in Uyo, a community in the Niger Delta Region, Nigeria. Health Care for Women International. 2010; 31(10):891-901. [PubMed: 20835939]

39. Devries KM, Kishor S, Johnson H, et al. Intimate partner violence during pregnancy: analysis of prevalence data from 19 countries. Reproductive Health Matters. 2010; 18(36):158-170. [PubMed: 21111360]

40. Kaye DK, Mirembe FM, Bantebya G, et al. Domestic violence as risk factor for unwanted pregnancy and induced abortion in Mulago Hospital, Kampala, Uganda. Tropical Medicine \& International Health. 2006; 11(1):90-101. [PubMed: 16398760]

41. Mbokota M, Moodley J. Domestic abuse-an antenatal survey at King Edward VIII Hospital, Durban. South African Medical Journal. 2003; 93(6):455. [PubMed: 12916387]

42. Makayoto LA, Omolo J, Kamweya AM, et al. Prevalence and associated factors of intimate partner violence among pregnant women attending Kisumu District Hospital, Kenya. Maternal and child health journal. 2013; 17(3):441-447. [PubMed: 22569943]

43. Guo S, Wu J, Qu C, et al. Physical and sexual abuse of women before, during, and after pregnancy. International Journal of Gynecology \& Obstetrics. 2004; 84(3):281-286. [PubMed: 15001384]

44. Saurel-Cubizolles M, Blondel B, Lelong N, et al. Marital violence after birth. Contraception, fertilite, sexualite (1992). 1997; 25(2):159.

45. Kanotra S, D'Angelo D, Phares TM, et al. Challenges faced by new mothers in the early postpartum period: an analysis of comment data from the 2000 Pregnancy Risk Assessment Monitoring System (PRAMS) survey. Maternal and child health journal. 2007; 11(6):549-558. [PubMed: 17562155]

46. O'Leary KD. Psychological abuse: A variable deserving critical attention in domestic violence. Violence and victims. 1999; 14(1):3-23. [PubMed: 10397623]

47. Campbell JC, Soeken KL. Forced Sex and Intimate Partner Violence Effects on Women's Risk and Women's Health. Violence against women. 1999; 5(9):1017-1035.

48. Countdown to 2015 Maternal, Newborn \& Child survival. World Health Organization. 2012.

49. Health SADo., editor. Guidelines for Maternity Care in South Africa. Third ed. Republic of South Africa; 2011.

50. Nelson HD, Bougatsos C, Blazina I. Screening Women for Intimate Partner Violence.

51. Kiely M, El-Mohandes AAE, El-Khorazaty MN, et al. An integrated intervention to reduce intimate partner violence in pregnancy: A randomized trial. Obstetrics and gynecology. 2010; 115(2 Pt 1):273. [PubMed: 20093899]

52. Tiwari A, Leung W, Leung T, et al. A randomised controlled trial of empowerment training for Chinese abused pregnant women in Hong Kong. BJOG: An International Journal of Obstetrics \& Gynaecology. 2005; 112(9):1249-1256. [PubMed: 16101604] 
53. Taft AJ, Small R, Hegarty KL, et al. Mothers' AdvocateS In the Community (MOSAIC)-nonprofessional mentor support to reduce intimate partner violence and depression in mothers: a cluster randomised trial in primary care. BMC public health. 2011; 11(1):178. [PubMed: 21429226]

54. Jones DL, Peltzer K, Villar-Loubet O, et al. Reducing the risk of HIV infection during pregnancy among South African women: A randomized controlled trial. AIDS Care. 2013; 25(6):702-709. [PubMed: 23438041]

55. Jewkes R, Nduna M, Levin J, et al. Impact of stepping stones on incidence of HIV and HSV-2 and sexual behaviour in rural South Africa: cluster randomised controlled trial. BMJ: British Medical Journal. 2008; 337

56. Kim JC, Watts CH, Hargreaves JR, et al. Understanding the impact of a microfinance-based intervention on women's empowerment and the reduction of intimate partner violence in South Africa. American Journal of Public Health. 2007; 97(10):1794. [PubMed: 17761566] 


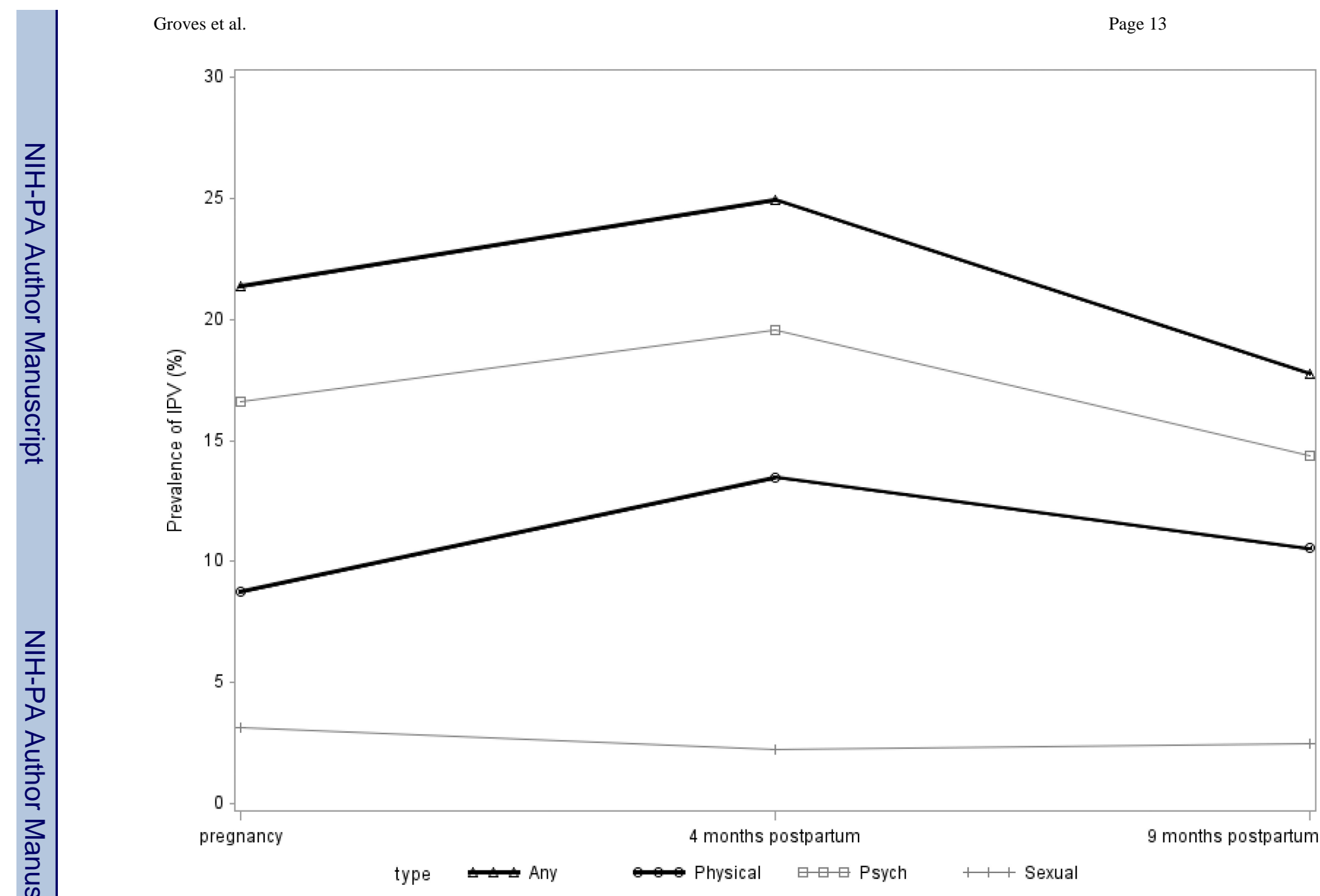

Figure 1.

Prevalence of IPV from pregnancy through nine months postpartum

Matern Child Health J. Author manuscript; available in PMC 2016 March 01. 
a.

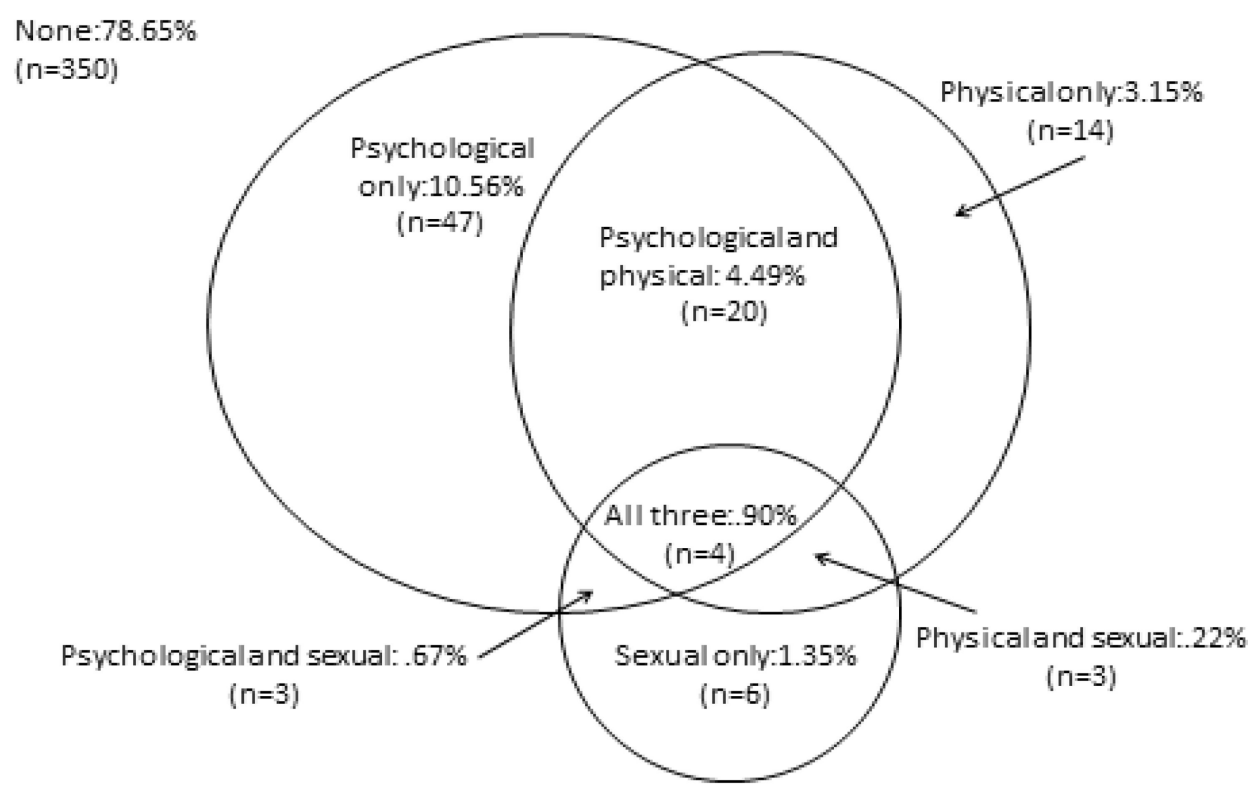

b.

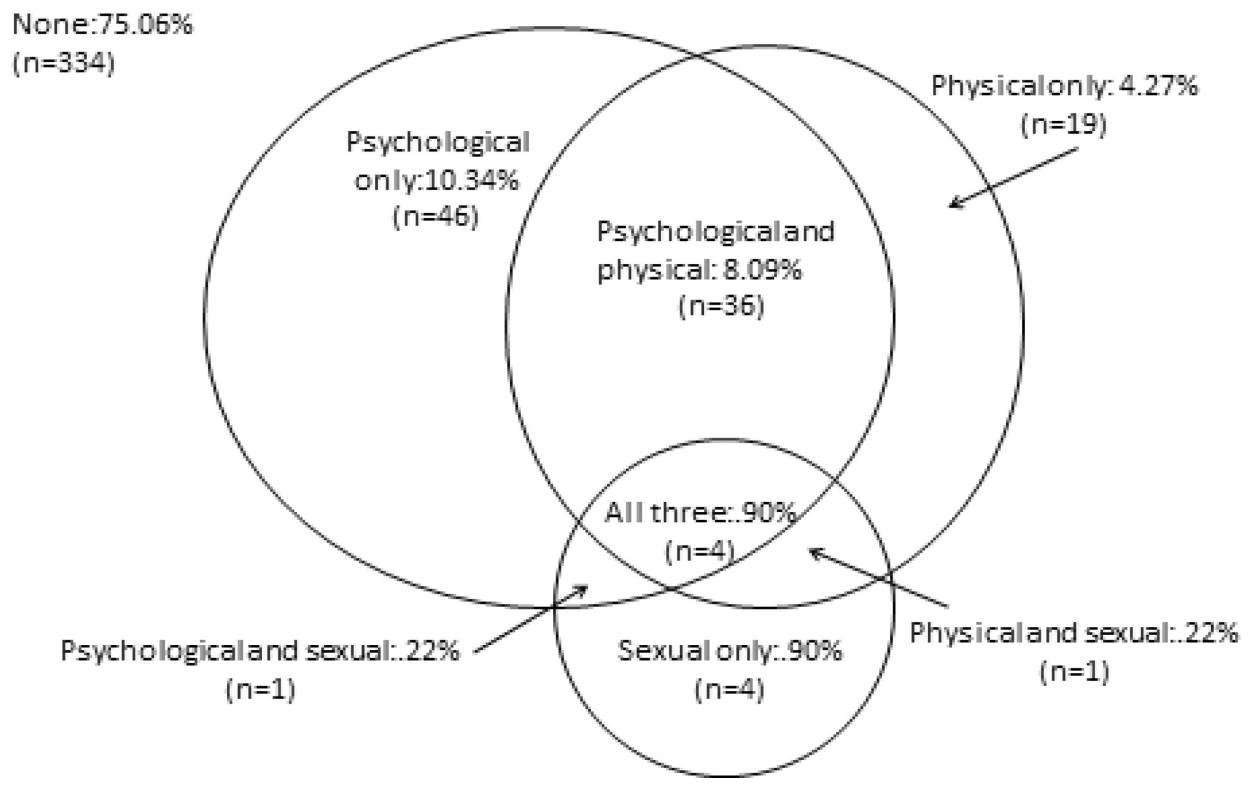

Matern Child Health J. Author manuscript; available in PMC 2016 March 01. 
c.

None:82.25\%
$(n=366)$

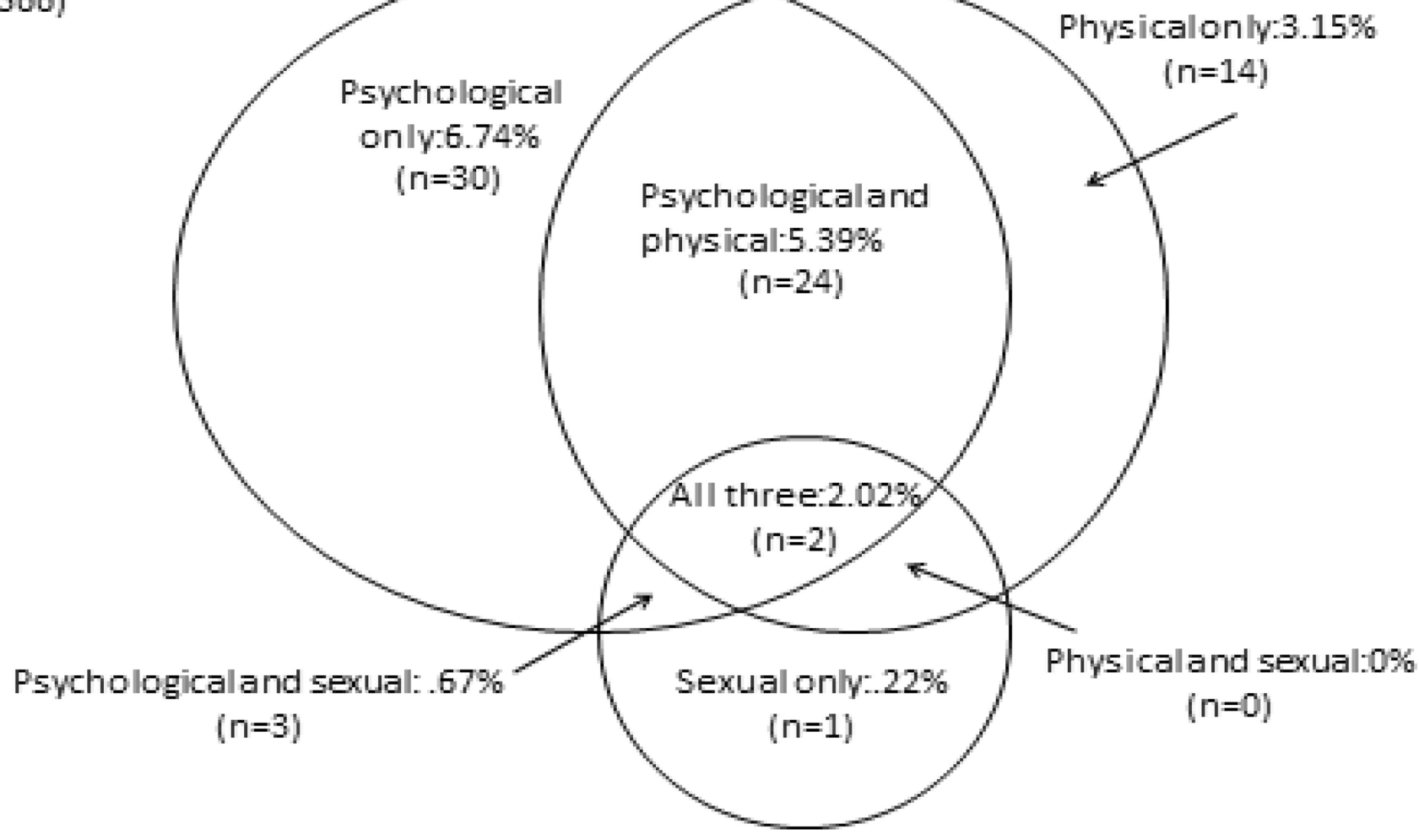

Figure 2.

Overlap in experiences of IPV during pregnancy and the postpartum period 


\section{Table 1}

Sociodemographic characteristics of the sample at baseline $(n=445)$

\begin{tabular}{|c|c|c|}
\hline & & (mean, sd) \\
\hline \multicolumn{2}{|l|}{ Participant's age (years) } & $25.89(5.80)$ \\
\hline \multicolumn{2}{|l|}{ Length of relationship (years) } & $4.71(4.29)$ \\
\hline \multirow{2}{*}{\multicolumn{2}{|c|}{ Gestational age (weeks) }} & $23.81(5.60)$ \\
\hline & & $(n, \%)$ \\
\hline \multicolumn{2}{|l|}{ Live together } & $112(25.17)$ \\
\hline \multirow[t]{3}{*}{ Education } & Primary & $27(6.07)$ \\
\hline & Secondary & $173(38.88)$ \\
\hline & More than secondary & $245(55.06)$ \\
\hline \multicolumn{2}{|l|}{$\mathrm{HIV}+$ status at baseline } & $158(35.51)$ \\
\hline \multirow[t]{3}{*}{ \# of prior pregnancies } & 0 & $164(36.85)$ \\
\hline & 1 & $161(36.18)$ \\
\hline & 2 or more & $120(26.97)$ \\
\hline Pregnancy unintended & & $350(79.73)^{*}$ \\
\hline
\end{tabular}




\section{Table 2}

Prevalence and rates of IPV during pregnancy and the postpartum period $(\mathrm{n}=445)$

\begin{tabular}{|c|c|c|c|}
\hline & During Pregnancy & $\begin{array}{l}\text { Delivery to four months } \\
\text { postpartum }\end{array}$ & $\begin{array}{l}\text { Four months to nine } \\
\text { months postpartum }\end{array}$ \\
\hline \multicolumn{4}{|l|}{ Prevalence estimates $^{a}$} \\
\hline Any IPV & $21.35(17.52,25.17)$ & $24.94(20.91,28.98)$ & $17.75(14.19,21.32)$ \\
\hline Physical IPV & $8.76(6.13,11.40)$ & $13.48(10.30,16.67)$ & $10.56(7.70,13.43)$ \\
\hline Psychological IPV & $16.63(13.16,20.10)$ & $19.55(15.85,23.25)$ & $14.38(11.11,17.66)$ \\
\hline Sexual IPV & $3.15(1.52,4.77)$ & $2.25(0.87,3.63)$ & $2.47(1.02,3.92)$ \\
\hline \multicolumn{4}{|l|}{ Rate estimates $b$} \\
\hline Any IPV rate & $0.15(.51)$ & $0.29(.89)$ & $0.27(1.57)$ \\
\hline Physical IPV rate & $0.05(.22)$ & $0.11(.40)$ & $0.12(.75)$ \\
\hline Psychological IPV rate & $0.09(.30)$ & $0.16(.46)$ & $0.12(.49)$ \\
\hline Sexual IPV rate & $0.02(.12)$ & $0.02(.21)$ & $0.03(.43)$ \\
\hline
\end{tabular}




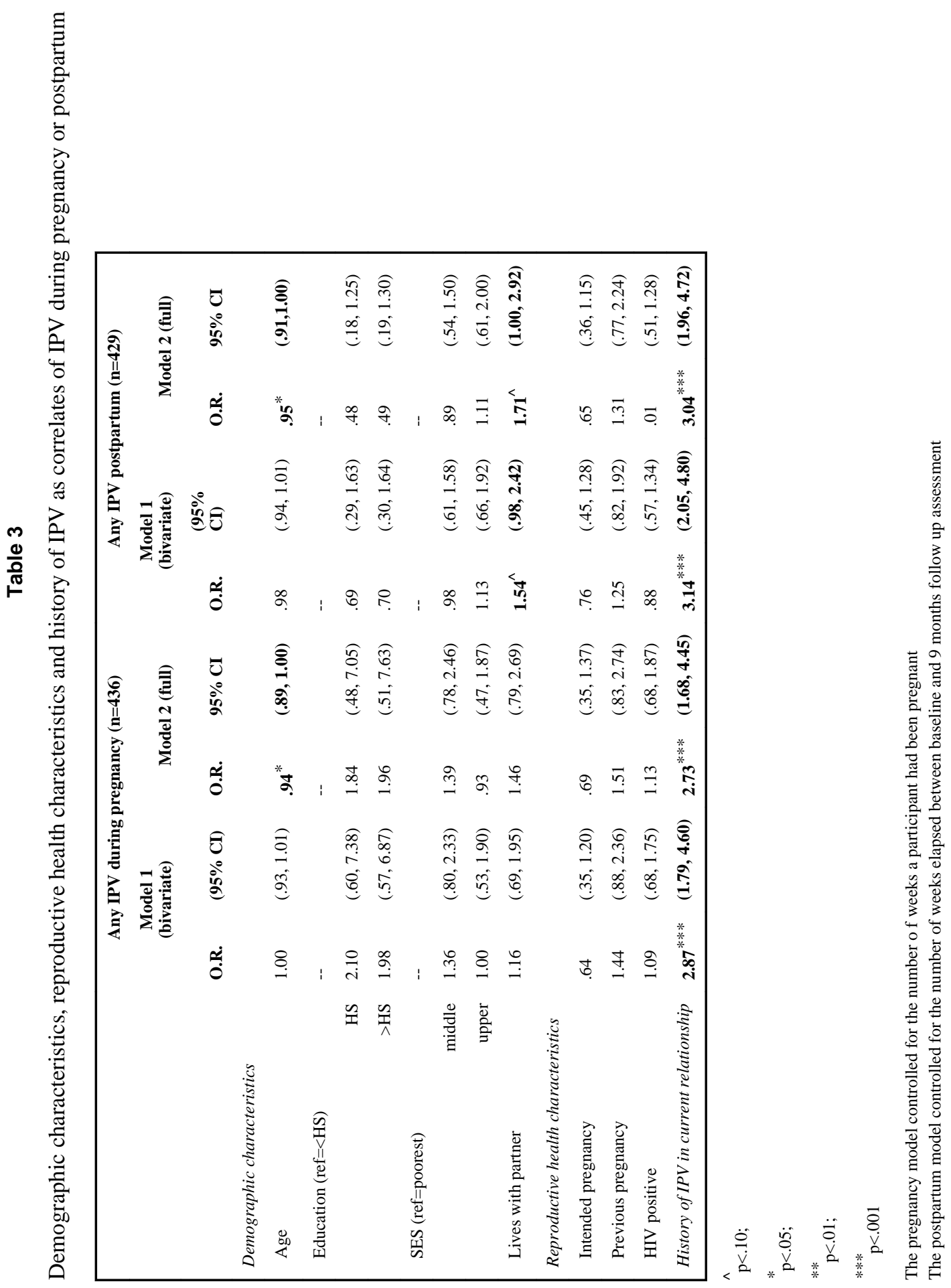

Matern Child Health J. Author manuscript; available in PMC 2016 March 01. 